\title{
Deprescribing in older adults: a new concept for nurses in administering medicines and as prescribers of medicine
}

\author{
Corina Naughton, ${ }^{1}$ Nicky Hayes ${ }^{2}$
}

\begin{abstract}
${ }^{1}$ Florence Nightingale Faculty of Nursing and Midwifery, Kings College London, London, UK

${ }^{2}$ Consultant Nurse for Older People, King's College Hospital NHS Foundation Trust, London, UK
\end{abstract}

\section{Correspondence to} Dr Corina Naughton, Florence Nightingale Faculty of Nursing and Midwifery, Kings College London, 57 waterloo Road, London SE1 8WA, UK; corina.naughton@kcl.ac.uk

$\mathrm{CN}$ and $\mathrm{NH}$ contributed equally to the writing of this manuscript.

Received 1 February 2016 Revised 3 May 2016 Accepted 4 May 2016

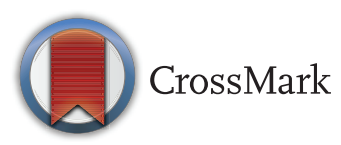

To cite: Naughton C, Hayes N. Eur J Hosp Pharm 2017;24:47-50.

\section{ABSTRACT}

Deprescribing is a new and emerging theme in the care of older adults living with multimorbidities including frailty. Deprescribing requires a comprehensive review of risk and benefits of a medication in the context of the quality of remaining life and patient and family priorities and preferences. Nursing to date has not engaged with this issue, yet in their roles administering medicines and prescribing medicines they are a fundamental part of the pathway in deprescribing decisions and in supporting patients to make such decisions. In administering medicines, nurses are in a position to observe the degree of difficulty or burden experienced by patients due to polypharmacy or side effects of medicines. While as prescribers for adults with multimorbidities, active review of the risk and benefits of all medicines using evidence-based instruments is part of prescribing responsibility. This article is calling for a critical examination of nurses' roles in deprescribing and in supporting patients to make informed choices about their treatment. There is a need to articulate the role of nursing in this emerging area of medicines management and contribute to a multidisciplinary discourse on deprescribing. Equally professional standards and continuous professional development for nurses as prescribers and administrators of medicines needs to reflect the complexity of an older population.

A deprescribing ethos challenges nurses to actively elicit patients' experiences of medicines burden, while nurse prescribers should recognise that appropriate deprescribing is as much a part of their role as appropriate prescribing.

\section{INTRODUCTION}

The concept of deprescribing is relatively new within medical discourse, it is even more novel within nursing literature. Deprescribing is defined as the "systematic process of identifying and discontinuing drugs in instances in which existing or potential harm outweigh existing or potential benefits within the context of an individual patients' goal, current level of functioning, life expectancy, values and preferences". ${ }^{1}$

The above definition outlines complex decision-making, whereby risks and benefits are weighed against quality of life, patient preferences and predictions of life expectancy. It is much more complex than stopping a medication due to unwanted side effects or substituting one therapy for another. To date, deprescribing and the implications for nursing practice as administrators of medicines and prescribers have not been explored.

\section{BACKGROUND}

In England, there has been a 55\% increase in the average number of items prescribed for each person per year, rising from 13.7 (2004) to 19.6 (2014). ${ }^{2}$ In addition, the rapidly expanding over-the-counter medicines market can add to the number of medicines taken. Unprecedented levels of polypharmacy, especially in the older-old population, combined with increases in patient harm and adverse drug events due to condition-drug and drug-drug interactions leading to morbidity and mortality are key drivers for a deprescribing movement. ${ }^{34}$

Deprescribing is an extension of the appropriate and rational prescribing ethos. It takes good prescribing practice (effective, safe, minimise risk, costeffective, respect for patient choices) and medicines optimisation principles one step further. ${ }^{56}$ There is an explicit emphasis on stopping medication in consultation with the patient and family rather than an isolated prescriber decision. Scott et al ${ }^{1}$ outlined a five-step deprescribing protocol. Step three in the protocol calls for the assessment of each drug for a deprescribing decision. Many of the cues considered in the deprescribing protocol are already part of established safe prescribing practice such as no valid drug indication, imbalance between harm versus benefit, therapy is ineffective or symptoms have resolved. Two of the deprescribing prompts have particular relevance for intimate nursing observation and communication with patients: (a) the preventive drug is unlikely to confer any patient-important benefit over the patients remaining life span and (b) drugs are imposing unacceptable treatment burden. ${ }^{1}$ Implementation of this detailed deprescribing process requires a sophisticated knowledge of pharmacology and high-risk drugs in the older population. But an equally important feature of the protocol is that in one-third of the 34 implementation guidance 'Ask' or 'Communicate' to the patient (and cares) is central to reaching a deprescribing decision. ${ }^{1}$ Deprescribing involves a comprehensive assessment of the person including their priorities and preferences rather that a quick 'crossing off' of a drug from a prescription.

With increased prevalence of long-term conditions including frailty, polypharmacy and improved scientific knowledge of ageing physiology and drug metabolism, deprescribing is becoming a more widespread concept. ${ }^{7}$ The nursing profession by virtue of a long-standing role and responsibility in drug administration and more recent role extension to medicines prescribing have an important contribution to developing the evidence base, skills and 
competencies for deprescribing. This paper examines the context of deprescribing from the perspective of nurses in medicines administration and prescribing practices and outlines the nature of the nursing contribution to this emerging topic.

\section{NURSE DRUG ADMINISTRATION}

A core nursing activity is the safe administration of medicines, which is one of the distinguishing responsibilities of Registered Nurses. In European Union countries, the equivalent regulatory bodies to the Nursing and Midwifery Council (NMC) in the UK set and uphold Standards for Medicines Management. ${ }^{8}$ The NMC standards stipulate 'It is not solely a mechanistic task...it requires thought and the exercise of professional judgement. ${ }^{8}$ The NMC standards recognise nurses' professional judgement in identifying adverse drug reactions, recording reasons for medicine refusal or 'not given' and ongoing monitoring for patients who self-administer medicine. The standards stop short of explicitly describing a role for nurses in contributing to deprescribing decisions.

However, nurses frequently witness what Scott $e t a l^{1}$ describe as the 'unacceptable treatment burden' associated with polypharmacy or individual drugs for some patients, especially those living with high levels of frailty. This is more than drug side effects, it describes the difficulty a patient may have in swallowing tables, the sheer volume of tables taken at a single point in time, handling difficulties such as opening containers, health beliefs, expense to patients or monitoring requirements (eg, warfarin sodium). This is vividly illustrated on the 08:00 drug round in acute care older adult units. The average number of medicines (tablet and liquid) per patient is 6-8 medicines, a frail older person may be expected to take these medicines, before breakfast and without much discussion. The nurses administering the medicines are under pressure to complete the drug round, which can typically take two registered nurses, working independently between 90 and $120 \mathrm{~min}$ by the time the last medicine is given. Nurses are faced with competing demands of administering large numbers of medication at the scheduled time while meeting the physical and emotional needs of very dependent people. If a patient refuses a medicine, this is recorded but often not the reason for refusing. While other types of medicine taking burden (eg, difficulty swallowing, dry mouth, nausea, poor appetite, residual drowsiness from night sedation) may not be systematically captured or relayed to the pharmacist or medical team.

The nurses may consider strategies to reduce this burden, such as crushing medicines (in itself carrying additional risk and considered in exceptional circumstances rather than routine practice). ${ }^{9}$ The alternative, deprescribing to reduce the number of medicines should be actively considered beyond the narrow context of drug side effects. There are even greater barriers for nurses working in nursing or residential settings, where access to medicines advice and reconciliation is more limited. ${ }^{10}$

\section{Capturing drug burden}

Active assessment of the medication taking burden experienced by older people may not be routine practice. Patients or carers may not be actively questioned about which medicines they find difficulty to take, medication regimes that cause confusion (eg, the variable warfarin dosing) or medications associated with milder side effects that a patient has learnt to live with or may not realise the symptoms are due to medication. In acute care settings, patient self-administration of medicines (SAM) programmes include detailed assessment of patients' ability to manage their medicines, which provides an opportunity to address medication taking burden. A Cochrane Review concluded that there is insufficient evidence to support the use of self-administration programmes to improve medicines adherence, knowledge, errors or satisfaction. ${ }^{11}$

There are instruments designed to capture the concept of medication burden, such as the Drug Burden Index, but it has a specific focus on anticholinergic and sedation medication. The Drug Burden Index calculates an individual's total exposure to these high-risk medications and calculates the risk of drug-related physical function decline. ${ }^{12}$ However, for many older people, it can be the low-risk drugs that are problematic, for example, macrogol compounds (unless swallowed quickly form into a thick paste), or other liquid/soluble preparations that leave an astringent taste reducing food enjoyment and intake. Dry mouth (xerostomia) is another common adverse side effect of medications (e.g. diuretics, aspirin), again contributing to reduced appetite and undernutrition in an already frail person. ${ }^{13}$

The issues related to medicine administration are important topics for further nurse-led research into recognition of medicine burden. This includes how medication burden is recognised, how this information influences nurses' decision-making and how such observations are recorded and relayed to the multidisciplinary team? A standard and validated risk assessment of medication burden could help contribute to deprescribing decisions, especially in adults with impaired cognitive ability or living with severe frailty. However, an assessment tool is not a substitute for effective communication between the nurse and patient and family or carers.

Patients are often reluctant to 'bother' nurses and will put up with quite high levels of discomfort before difficulty with medication taking becomes obvious. The pharmacological interactions and major side effects, though very important, underrecognises the burden of drug taking in an older more frail population. Increased awareness and active questioning by the nursing team should be standard practice and include questions on dry mouth, difficulty swallowing, nausea or poor appetite. A simple screening question could be: Are there any of your medicines that you do not like or have difficulty taking regularly (and why)? Nursing's biopsychosocial approach is well placed to explore patients' health beliefs and the influence these have on medication taking behaviours. Such questions could be part of a patient admission process with regular review during an episode of care. Nurses tend to leave decisions on medication reconciliation or deprescribing to other members of the multidisciplinary team and may not recognise they are in an important position to contribute valuable observations and insights to these decisions.

\section{NURSE PRESCRIBERS}

The number of countries introducing and expanding nurse prescribing is increasing. ${ }^{14} \mathrm{~A}$ systematic review compared physician and nurse prescribing and concluded that nurse prescribing was similar to physicians in terms of number of patients and range of drugs and doses prescribed. ${ }^{15}$ Patient and clinical outcomes were also similar though there was a tendency towards higher patient satisfaction with nurse prescribers. ${ }^{15}$ The strength of this evidence is limited by a lack of well-conducted randomised controlled trials and the inclusion of quite dated studies (>10 years).

Although the principles of deprescribing are relevant to nurse prescribing practice, a search of the literature has identified no articles specifically examining nurse deprescribing practice. Even evaluation of nurse prescribing in older people is limited. 
Secondary analysis of a population database of drug prescribing in Ontario compared trends in nurse prescribing in older adults (>65 years) with family physicians over a 10 year period. ${ }^{16}$ There was a substantial increase in nurses prescribing for older adults from $12 \%$ in 2000 up to $62 \%$ in 2010 , however, considerable regional variability was noted in nurses' prescribing. There was a significant change in the pattern of prescribing with a shift in nurses mainly prescribing for acute conditions in 2000 (eg, antibiotics) to prescribing for chronic conditions $(9 / 10$ of the most frequently prescribed drugs were used in chronic disease management). ${ }^{16}$ This pattern of drug prescribing was largely similar to that of the comparator group of family physicians with statins, antihypertensives and proton-pump inhibitors being the most frequently prescribed drugs by both groups of prescribers. ${ }^{16}$ These findings confirmed an earlier self-report survey of gerontological nurse practitioners where the majority of prescribing was for chronic conditions and pain management. ${ }^{17}$ Deprescribing practice was not examined, but it did look at inappropriate prescribing using the Beers Criteria. Approximately $25 \%$ of nurses reported regular use of at least one of the 29 high-risk drugs listed on the Beers Criteria. ${ }^{18}$ While there is a growing body of research on nurse prescribing for chronic conditions, research on nurse prescribing for people with multimorbidity is limited, equally nurses use of medication reconciliation tools such as the screening tool of older people's prescriptions (STOPP) and screening tool to alert to right treatment (START) criteria to inform prescribing decisions in older adults is absent from the literature. ${ }^{19}$

\section{COMPETENCIES OF NURSE PRESCRIBERS IN OLDER ADULTS}

In the UK, the competencies for nurse prescribers are laid out by the NMC and contain 21 practice standards for nurse and midwife prescribing. ${ }^{20}$ Unlike children and young adults, there are no specific prescribing competencies for older adults. Yet there is growing evidence of the physiological and pharmacokinetic complexity associated with the ageing process. In addition, there is sometimes the need for consideration of the legal, cognitive, emotional and physical differences of prescribing in older adults. The complexities of prescribing in older adults illustrate the need for expanded competencies for prescribing and deprescribing in this population. ${ }^{21}$ Deprescribing should be part of the normal drug prescribing pathway, whereby any medication, including those for long-term conditions, have a therapeutic 'end date'. ${ }^{22}$

Nurse prescribers can be bounded by a focus on specific conditions and may lack the broader knowledge and skills to prescribe for older adults living with multimorbidities and frailty. ${ }^{23} 24$ Contemporary approaches to interprofessional assessment and treatment planning for older people indicate that frailty screening and application of Comprehensive Geriatric Assessment (CGA) provides an appropriate framework within which to address the complexities of medicines management. ${ }^{25}$ However, this has yet to gain common currency within nursing. There are less and less nurses in the workforce gaining specialist qualifications in older person's nursing due to limited availability of specific postgraduate courses and a lack of prioritisation within the National Health Service to develop specialist older person's nurse practitioners. ${ }^{26}$ The unique challenges in prescribing in older adults can be underestimated by a nurse workforce who often lacks specific older person's competencies and skills. ${ }^{27}$ This is not a problem limited to nurse prescribers and mirrors the discourse for the development of expanded competencies for medical prescribers. ${ }^{21}{ }^{28}$ Generic nurse prescribing courses provide the foundation for nurse prescribing; however, without specialist training nurses tend to limit their prescribing activity to specific conditions rather than across multiple conditions. ${ }^{24} 29$

It can be argued that nurses prescribing for older people (especially the older old) need to manage complex decisionmaking underpinned by a CGA approach, requiring broader and higher level expertise. Such expertise is difficult to gain through routine continuous professional development (CPD). A qualitative study with seven community matrons, working mainly with older adults living with complex health and social needs, described the importance of their prescribing in meeting patients' needs. ${ }^{30}$ However, the group were also conservative in their practice, restricting prescribing mainly to exacerbations of chronic obstructive pulmonary disease and antibiotics for infections; prescribing in conditions such as heart failure were avoided and none of the participants alluded to deprescribing medication started by another prescriber. ${ }^{30}$ The group highlighted challenges in receiving consistent high-quality $\mathrm{CPD}$ designed to expand their range of prescribing and professional competencies. ${ }^{30}$

One final consideration is that deprescribing can be narrowly considered in the context of palliative care, whereby drugs are discontinued in the immediate days before a person dies. Deprescribing is relevant at a much earlier stage and can be part of wider discussions on a person's priorities and preferences as part of models of CGA and advanced care planning. Such patient consultations require skill and confidence to negotiate the risk and benefits of medicines in the context of the remaining quality of life with the older person and the family central to the decision-making. Yet these skills can be undervalued, a survey of Advanced Nurse Practitioners ranked such skills (assess client healthcare risk, evaluate own practice for continuous improvement, assess clients therapeutic self-management, adapts communication style to meet needs of client, demonstrates effective working relations with healthcare team) as the least important of 31 competencies for nurse prescribers. ${ }^{31}$

The limited evidence on deprescribing competencies suggests there needs to be a cultural shift in preparing all healthcare professionals with prescriptive authority. Although deprescribing is regarded as part of the normal prescriptive pathway, it is not routine practice for junior prescribers to actively deprescribe. ${ }^{21}$ Even senior prescribers are likely to more actively deprescribe and reduce doses of medication prescribed by 'experts' when given specific tools and prompts. ${ }^{32}$ The foundation preparation of nurse prescribers and ongoing CPD need to be cognisant of developments in this area of practice and actively support nurses to develop confidence and competence to appropriately deprescribe in patients with comorbid therapies and to reflect an individual's changing priorities and preferences as they age.

\section{CONCLUSION}

Deprescribing is not yet integrated into either nurse medicine administration or nurse prescribing, yet nurses are often fundamentally involved in supporting older people's concordance with medicines. Deprescribing for older people involves complex decision-making, understanding of the ageing process and skilled negotiation combined with knowledge of the pharmacological and physical burden of medication taking. Central to this decision is communication with the older person and the family. Practitioners working with older people living with high levels of comorbidities, polypharmacy and frailty need support and CPD to develop advanced skills and competencies, applying deprescribing principles within a CGA 
framework. Medicines reconciliation and deprescribing tools may help support decision-making. There is also a role for regulatory bodies responsible for standards in medication administration and prescribing to work with education providers and frontline practitioners to ensure deprescribing becomes normative practice in drug prescription pathways.

Twitter Follow Corina Naughton at @corinanaughton1

Collaborators Barry Jubraj.

Competing interests None declared.

Provenance and peer review Not commissioned; externally peer reviewed.

\section{REFERENCES}

1 Scott IA, Hilmer SN, Reeve E, et al. Reducing inappropriate polypharmacy: the process of deprescribing. JAMA Intern Med 2015;175:827-34.

2 NHS Information Centre. Prescriptions Dispensed in the Community: England, Statistics for 2004 to 2014 NHS website. 2015. http://www.hscic.gov.uk/catalogue/ PUB17644/pres-disp-com-eng-2004-14-rep.pdf (accessed 20 May 2016).

3 Cousins DH, Gerrett D, Warner B. A review of medication incidents reported to The National Reporting and Learning System in England and Wales over 6 years (20052010). Br J Clin Pharmacol 2012;74:597-604.

4 Tommelein E, Mehuys E, Petrovic M. Potentially inappropriate prescribing in community-dwelling older people across Europe: a systematic literature review. Eur J Clin Pharmacol 2015;71:1415-27.

5 Payne R, Abel G, Avery A. Is polypharmacy always hazardous? A retrospective cohort analysis using linked electronic health records from primary and secondary care. Br J Clin Pharmacol 2014;77:1073-82.

6 Royal Pharmaceutical Society. Medicines Optimisation: Helping patients to make the most of medicines. 2013. https://www.rpharms.com/promoting-pharmacy-pdfs/ helping-patients-make-the-most-of-their-medicines.pdf (accessed 1 Feb 2016).

7 Hubbard RE, O'Mahony MS, Woodhouse KW. Medication prescribing in frail older people. Eur J Clin Pharmacol 2013;69:319-26.

8 Nursing and Midwifery Council. Standards for Medication Management. NMC, 2010. http://www.nmc.org.uk/standards/additional-standards/ standards-for-medicines-management/ (accessed 14 Dec 2015).

9 Bourdeneta G, Giraudb S, Artura M. Impact of recommendations on crushing medications in geriatrics: from prescription to administration. Fundam Clin Pharmacol 2015;29:316-20.

10 Fahrni ML, Franklin BD, Rawaf $S$, et al. Improving medication safety in UK care homes: challenges and current perspective. JRSM Open 2014:5:2042533313515475.

11 Ryan R, Santesso N, Lowe $D$, et al. Interventions to improve safe and effective medicines use by consumers: an overview of systematic. Cochrane Database Syst Rev 2014;4:CD007768.

12 Kouladjian L, Gnjidic D, Chen TF, et al. Drug Burden Index in older adults: theoretical and practical issues. Clin Interv Aging 2014;9:1503-15. eCollection 2014.
13 Thomson M, Chalmers JM, Spencer J, et al. A longitudinal study of medication exposure and xerostomia among older people. Gerodontology 2006;23:205-13.

14 Kroezen M, Francke AL, Groenewegen PP, et al. Nurse prescribing of medicines in Western European and Anglo-Saxon countries: a survey on forces, conditions and jurisdictional control. Int I Nurs Stud 2012;49:1002-12.

15 Gielen SC, Dekker J, Francke AL, et al. The effects of nurse prescribing: a systematic review. Int J Nurs Stud 2014;51:1048-61.

16 Tranmer JE, Colley L, Edge DS, et al. Trends in nurse practitioners' prescribing to older adults in Ontario, 2000-2010: a retrospective cohort study. CMAJ Open 2015;3:E299-304.

17 Kennedy-Malone L, Fleming ME, Penny J. Prescribing patterns of gerontological nurse practitioners in the United States. J Am Acad Nurse Pract 2008;20: 28-34.

18 Beers $\mathrm{M} \mathrm{H}$. Explicit criteria for determining potentially inappropriate medication use by the elderly. Arch Intern Med 1997;157:1531-6.

19 O'Mahony D, O'Sullivan D, Byrne S, et al. STOPP/START criteria for potentially inappropriate prescribing in older people: version 2. Age Ageing 2015;44: 213-18.

20 NMC Standards of proficiency for nurse and midwife prescribers. NMC, 2006. http://www.nmc.org.uk/standards/additional-standards/ standards-of-proficiency-for-nurse-and-midwife-prescribers/

21 Jubraj B, Marvin V, Poots A, et al. A pilot survey of junior doctors' attitudes and awareness around medication review: time to change our educational approach? Eur J Hosp Pharm Sci Pract 2015;22:243-8.

22 Iyer S, Naganathan V, McLachlan AJ, et al. Medication withdrawal trials in people aged 65 years and older: a systematic review. Drugs Aging 2008;25:1021-31.

23 Richmond J. Non-medical prescribing for the older population. J Diabetes Nur 2004;19:180-1.

24 Carey N, Stenner K, Courtenay M. An exploration of how nurse prescribing is being used for patients with respiratory conditions across the east of England. BMC Health Serv Res 2014;14:27.

25 British Geriatrics Society. Fit for Frailty. 2014. http://www.bgs.org.uk/campaigns/fff/ fff_full.pdf (accessed 31 Jan 2016).

26 Royal College of Nursing. Safe staffing for older people's wards. 2012. https://www. rcn.org.uk/_data/assets/pdf_file/0009/476379/004280.pdf (accessed 17 Dec 2015).

27 Williams B, Warshaw G, Fabiny A, et al. Medicine in the 21st century: recommended essential geriatrics competencies for internal medicine and family medicine residents. J Grad Med Educ 2010;2:373-83.

28 Pearson P, Steven A, Tiplady S, et al. The National Career Framework for nurses caring for older people with complex needs in England. Northumbria University, 2015. ISBN:978-0-9933289-0-9.

29 Stenner K, Carey N, Courtenay M. Prescribing for pain-how do nurses contribute? A national questionnaire survey. J Clin Nurs 2012;21:3335-45.

30 Herklots $A$, Baileff $A$, Latter $S$. Community matrons' experience as independent prescribers. Br I Community Nurs 2015;20:217-18, 220-3.

31 Klein T, Kaplan L. Prescribing competencies of advanced practice registered nurses. J Nurse Pract 2010:6:115-22.

32 Duraisingham S, Jubraj B, Marvin V, et al. Stopping inappropriate medicines in the outpatients setting. Gen Med 2015:35-41. 